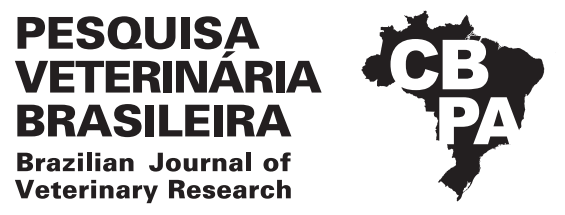

Pesq. Vet. Bras. 38(7):1449-1452, julho 2018 DOI: $10.1590 / 1678-5150-P V B-5344$

Original Article

Morfofisiologia/Animal Morphophysiology

ISSN 0100-736X (Print)

ISSN 1678-5150 (Online)

\title{
Telencephalon vascularity in sheep (Ovis aries) ${ }^{1}$
}

\author{
Benedykt Skoczylas², Witold Brudnicki², Krzysztof Kirkiłło-Stacewicz²*, \\ Włodzimierz Nowicki ${ }^{2}$ and Jan Wach ${ }^{2}$
}

\begin{abstract}
Skoczylas B., Brudnicki W., Kirkiłło-Stacewicz K., Nowicki W. \& Wach J. 2018. Telencephalon vascularity in sheep (Ovis aries). Pesquisa Veterinária Brasileira 38(7):1449-1452. Department of Animal Morphology and Hunting, Faculty of Animal Breeding and Biology, UTP University of Science and Technology, Bernardyńska 6/8, 85-029, Bydgoszcz, Poland. E-mail: anat@utp.edu.pl

The studies into the vascularity of the telencephalon in Polish Merino sheep of both sexes were made on 60 cerebral hemispheres. It was found that the middle cerebral artery is the most powerful vessel supplying blood to the telencephalon. The artery gets divided into ten permanent branches. Two olfactory arteries supply the area of the telencephalon located on the border between the old and the new cortex. The other eight branches get divided into three branches running to the region of the frontal lobus of the brain, two branches to the region of the parietal lobus and three temporal branches heading for the temporal region, supplying blood to the new cortex only. The frontal, parietal and temporal branches descended independently from the main trunk of the middle cerebral artery or first formed a common trunk. Common trunks for respective groups of branches have been referred to as: the anterior, superior and posterior middle cerebral arteries. The posterior olfactory artery in $6.7 \%$ of the cases was an independent branch from the rostral cerebral artery.
\end{abstract}

INDEX TERMS: Telencephalon, vascularity, sheep, Ovis aries, arteries, brain.

\section{INTRODUCTION}

The applicable literature on the vascularity of the brain in mammals provides reports the authors of which offer detailed descriptions of the cortical braches of the middle cerebral artery e.g. described in cat by Chadzypanagiotis (1975) as well as Wiland (1991) in some Carnivora species. In Artiodactyla there have been found some descriptions of cortical branches of the middle cerebral artery in domestic pig (Walinczus 1973), European bison (Węgrzyn et al. 1983), in roe deer (Jabłoński \& Roskosz 1997), in moose and cow (Jabłoński et al. 1999a, 1999b), in wild boar (Skoczylas \& Wiland 1999), in goat (Brudnicki et al. 2005), in fallow deer (Skoczylas et al.2011). Those reports show that the cortical branches of the middle cerebral artery in those animal species reach the same areas of the brain. The differences concern the pattern of descent and division of respective cortical branches of the middle cerebral artery. The division pattern of the middle cerebral artery is affected by various factors;

\footnotetext{
${ }^{1}$ Received on May 30, 2017.

Accepted for publication on October 16, 2017.

${ }^{2}$ Department of Animal Morphology and Hunting, Faculty of Animal Breeding and Biology, UTP University of Science and Technology, Bernardyńska 6/8, 85-029, Bydgoszcz, Poland. Tel. (052) 37495 65. *Corresponding author: krzysztof.stacewicz@o2.pl
}

e.g. the classification the species represents and the groove pattern of the cortex. In mammals on the surface of the cortex there occurs a varied groove pattern, which can affect the structure of the cortical branches of the middle cerebral artery (Brauer \& Schaber 1970). The vast literature, however, seems to be missing information on the cortical branches of the middle cerebral artery in sheep. One can, however, come across papers where the authors describe the pattern of blood supply to the brain in that species (Baldwin \& Bell 1963, Baldwin 1964) and the structure and variation in the arteries of the base of the brain (Jabłoński \& Wiland 1973). Considering the discrepancies resulting from respective descriptions as well as factoring in new reports, it has been decided to investigate the pattern, the pattern of division and variation in cortical branches of the middle cerebral artery in sheep and to compare the results with the data reported by other authors.

\section{MATERIALS AND METHODS}

Ethics statement. The experiment was conducted at Department of Animal Morphology and Hunting, UTP University of Science and Technology, Poland, in compliance with the Ethics Principles in Animal Experimentation, being approved by the Ethics Committee on Animal Experimentation (CEUA/CNPSA) (Protocol \#005/2010). 
Animals. The studies of the middle cerebral artery covered 30 Polish Merino sheep brains of both sexes. Adult animals were slaughtered, subjected to culling.

Study design. 60 cerebral hemispheres were analysed and sheep heads were cut off at the height of the 3rd-4th cervical vertebrae. Having prepared the common carotid arteries, one of them was entered into with the medical syringe needle and the arteries were filled with black-dyed latex. The heads were fixed in a $5 \%$ formalin solution for 3 months and then, having removed the muscles, the bones were decalcified in a $5 \%$ hydrochloric acid. Then the skull cavity was opened and the brain was taken out. The cerebral hemispheres were photographed and the anatomy, the division and the pattern of the cortical branches of the middle cerebral artery were described.

Statistics. Descriptive analysis was performed and the overall result shown in figures.

\section{RESULTS}

The brain in sheep is supplied with blood through internal carotid arteries the intracranial section of which gets recovered from the rostral epidural rete mirabile. The extracranial section of the internal carotid artery undergoes atrophy after birth. Having passed the dura mater, the internal carotid artery gets divided into the rostral cerebral artery and caudal communicating artery which, together with the symmetrical vessels, form the arterial circle of the brain. At the height of the rostral border of the optic chiasma, the rostral cerebral artery gives off a thick arterial vessel; the middle cerebral artery. The middle cerebral artery is the most powerful vessel supplying blood to the telencephalon. The initial section of the main trunk of the middle cerebral artery goes along the ventral surface of the optic tract. Then the section bends around the piriform lobe and runs before its rostral border. Further on it runs towards the lateral rhinal sulcus and then, having passed it, it gets divided. From the initial section of the main trunk of the middle cerebral artery there descend minor central branches supplying blood to olfactory tracts and the piriform lobe. The main trunk of the middle cerebral artery gets divided into a number of cortical branches which run towards the specific region of the cerebral hemisphere, supplying blood to specific regions of the brain.

The first permanent branches of the middle cerebral artery which supply both the old and the new cortex are olfactory arteries.

The anterior olfactory artery (Fig.1.1), having separated from the main trunk of the middle cerebral artery, runs towards the rostral part of the lateral rhinal sulcus it can descend into in various places. Its terminal branches can also appear again from under the lateral rhinal sulcus and they ascend under the surface of the cortex.

The posterior olfactory artery (Fig.1.2) ascends into the caudal part of the lateral rhinal sulcus and its terminal branches supply blood also to the area of the cortex located over that sulcus. The other branches of the middle cerebral artery supply blood to the areas of the cortex found over the lateral rhinal sulcus. On the cortex, towards the frontal lobus, there spread three thick branches.

The orbital branch descends first (Fig.1.3); it supplies blood to the area of the cortex situated over the presylvian sulcus and below the diagonal sulcus. Its terminal branches reach the coronary sulcus.

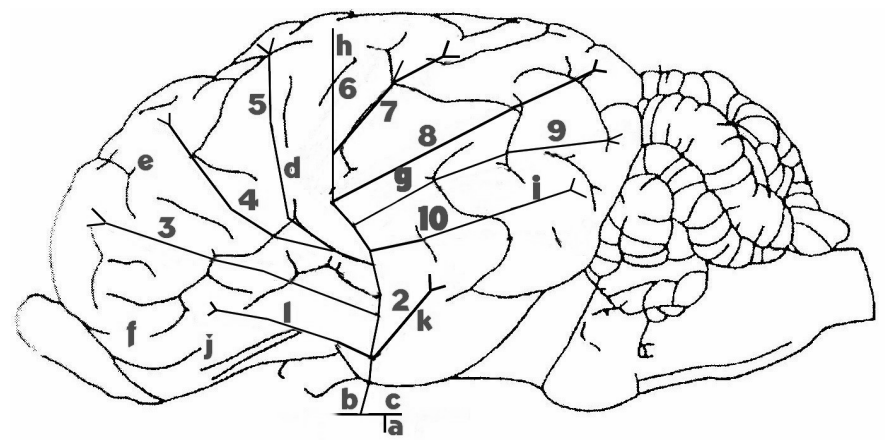

Fig.1. Division pattern of the middle cerebral artery on the surface of the cortex in sheep. Anterior olfactory artery (1), posterior olfactory artery (2), orbital branch (3), inferior frontal branch (4), superior frontal branch (5), anterior parietal branch (6), posterior parietal branch (7), superior temporal branch (8), middle temporal branch (9), inferior temporal branch (10); internal carotid artery (a), rostral cerebral artery (b), caudal communicating artery (c), Sylvian fissure (d), diagonal sulcus (e), presylvian sulcus (f), posterior external Sylvian sulcus (g), medial suprasylvian sulcus (h), caudal suprasylvian sulcus (i), rostral lateral rhinal sulcus (j), caudal lateral rhinal sulcus $(\mathrm{k})$.

The inferior frontal branch (Fig.1.4) runs towards the diagonal sulcus and then one of its branches ascends into that sulcus and the others spread on the surface of the cortex between the ansiform sulcus and the middle suprasylvian sulcus.

The superior frontal branch (Fig.1.5), having descended from the main trunk of the middle cerebral artery, ascends into the initial section of the middle suprasylvian sulcus. Its terminal branches supply blood to the upper part of the medial surface of the frontal lobus.

Another vessel which runs towards the parietal lobus ascending to the Sylvian fissure gets onto the surface of the cortex and after a short course it bifurcates into two branches.

The anterior parietal branch (Fig.1.6) and posterior parietal branch (Fig.1.7); the vessels run towards the middle suprasylvian sulcus. Having passed the sulcus, the vessels spread medially reaching the marginal sulcus. The lateral-posterior surface of the hemisphere is supplied by the branches of the middle cerebral artery which descend at various heights and they have been referred to as temporal branches.

The superior temporal branch (Fig.1.8) is usually the strongest cortical branch of the middle cerebral artery and its further prolongation in the cortex area.

Having descended from the Sylvian fissure, it goes towards the caudal suprasylvian sulcus. Then, having passed the marginal sulcus, its terminal branches reach the internal marginal sulcus they do not cross any more.

The middle temporal branch (Fig.1.9) descends in a little distance from the previous branch. It runs over the posterior external Sylvian sulcus. The terminal branches run towards the terminal section of the caudal suprasylvian sulcus and they get onto the surface of the occipital lobus.

The inferior temporal branch (Fig.1.10), having separated from the main trunk of the middle cerebral artery on the surface of the cortex, supplies blood to the area between the lateral rhinal sulcus and the posterior external Sylvian sulcus.

Its terminal branches participate in the supply of a part of the occipital lobus. 
Considering the presented general pattern of the distribution of cortical branches of the middle cerebral artery, one shall note that respective sections of those branches can get located inside respective sulci, sometimes undergoing further divisions, always going I the direction of the area of the cortex described.

Factoring in the pattern of descent of cortical branches of the middle cerebral artery in the sheep individuals, it was found that in 56 (93.3\%) cases from the rostral cerebral artery there descended one independent vessel; the middle cerebral artery. Among them, on $10(16.7 \%)$ hemispheres from the main trunk of the middle cerebral artery there descended rostrally a common trunk for the anterior olfactory artery, for the orbital branch and for the inferior and superior frontal branches. The main trunk caudally separated an independent posterior olfactory artery, then a common descent for the temporal branches: inferior, middle and superior as well as a common descent for parietal branches.

In 14 (23.3\%) cases from the main trunk there descended rostrally an independent anterior olfactory artery, followed by a common descent for the orbital branch and for inferior and superior frontal branches. Caudally, with a common trunk, from the main trunk there separated, the middle and inferior temporal branches as well as the posterior olfactory artery. The main trunk, having ascended into the Sylvian fissure, brought a common descent for anterior and posterior parietal branches as well as the superior temporal branch into the surface of the cortex.

On another $8(13.3 \%)$ hemispheres from the main trunk there separated rostrally, with a common descent, the orbital branch, the inferior frontal branch with the anterior olfactory artery. The main trunk of the middle cerebral artery, having brought onto the surface of the cortex, separated a common trunk for the superior frontal branch and anterior and posterior parietal branches. Caudally from the main trunk there descended, with a common descent, the temporal branches: inferior, middle and superior as well as an independent posterior olfactory artery.

In another $10(16.7 \%)$ cases from the main trunk of the middle cerebral artery rostrally there descended a common trunk for the anterior olfactory artery and for the orbital branch, then a common descent for the inferior and superior frontal branches. The main trunk caudally separated the posterior olfactory artery with a common descent from the inferior temporal branch. Having ascended into the Sylvian fissure, a common trunk for anterior and posterior parietal branches as well as for the middle and superior temporal branches got onto the surface of the cortex.

On the other $6(10.0 \%)$ hemispheres rostrally from the main trunk of the middle cerebral artery there descended independently the anterior olfactoral artery, then the common trunk for the orbital branch and the inferior frontal branch. Caudally from the main trunk there separated an independent posterior olfactory artery as well as a common descent for temporal branches. The main trunk, having ascended into the Sylvian fissure, brought a common descent for the superior frontal branch and for the parietal branches onto the surface of the cortex (Fig.2).

In the other $4(6.7 \%)$ cases it was found that from the rostral cerebral artery there bifurcated two independent branches of the middle cerebral artery. The first independent

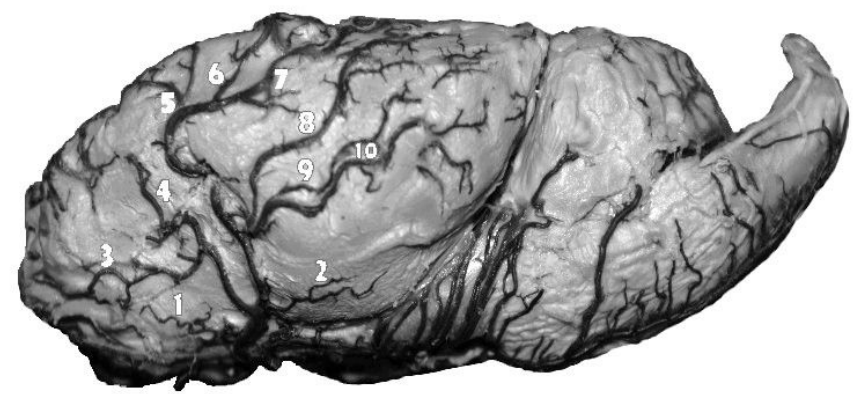

Fig.2. Single trunk of the middle cerebral artery and the respective cortical branches. Anterior olfactory artery (1), posterior olfactory artery (2), orbital branch (3), inferior frontal branch (4), superior frontal branch (5), anterior parietal branch (6), posterior parietal branch (7), superior temporal branch (8), middle temporal branch (9), inferior temporal branch (10).

descent from the rostral cerebral artery was the posterior olfactory artery, while the second descend from the rostral cerebral artery was the main trunk of the middle cerebral artery which rostrally gave rise to, with a common descent, the anterior olfactory artery, the orbital branch, inferior and superior frontal branches as well as parietal branches. Caudally from the main trunk there separated temporal branches with a common trunk.

\section{DISCUSSION}

In sheep the middle cerebral artery supplies the same areas of the brain as in the mammal species studied so far. The discrepancies concern mostly its division into respective branches. Chadzypanagiotis (1975), describing the cortical branches in cat, differentiated the branches supplying the old cortex, the branches on the border of the old and the new cortex as well as the branches for the new cortex. In sheep the arteries supplying the old cortex are minor arterial vessels onto the piriform lobe, perforated body and olfactory tracts. On the border of the old and the new cortex there run anterior and posterior olfactory arteries. In sheep the anterior olfactory artery in $30 \%$ of the cases investigated was a vessel which descended independently from the main trunk of the middle cerebral artery. In the other hemispheres it was a vessel which got differentiated with a common trunk with the orbital branch or common descent with the orbital, inferior and superior frontal branches. The same form of descent of the anterior olfactory artery was dominant in goat (Brudnicki et al. 2005), while the posterior olfactory artery in $6.7 \%$ cases was a vessel which descended directly from the rostral cerebral artery. In the other hemispheres in $36.7 \%$ of the cases it was a vessel descending from the main trunk or it was one of the branches of the common trunk for temporal branches and directly descended from the inferior temporal branch.

The other cortical branches of the middle cerebral artery can be divided into a group of frontal, parietal and temporal branches. In sheep, similarly as in other ruminant species, there occur eight main vessels which supply blood to the same areas of cerebral hemispheres. Besides, respective cortical branches can descend from the main trunk of the middle cerebral artery with a common descent. Such cases 
of descent were described by Wiland (1991) as the anterior, superior and posterior middle cerebral artery. In sheep the anterior middle cerebral artery has been demonstrated as a common trunk for frontal branches and it occurred in 23.3\% of the cases investigated, the superior middle cerebral artery referred to as a common trunk for parietal branches accounted for $16.7 \%$ of the cases. The posterior middle cerebral artery as a common trunk for temporal branches accounted for $36.7 \%$ of the cases. In sheep the superior middle cerebral artery was reported at the lowest percentage of the cases, while here the posterior middle cerebral artery dominated. Comparing the results with those reported by Jabłoński et al. (1999b), one can observe that also in cattle the posterior middle cerebral artery accounted for the highest percentage of the cases.

In sheep, similarly as in Artiodactyla species investigated, the parietal branches are least developed. On the surface of the telencephalon the temporal branches of the middle cerebral artery are best developed.

The descriptions of the anatomy of the middle cerebral artery provided by Brudnicki et al. (2005), Skoczylas et al. (2011, 2012), in red deer, goat, fallow deer and European otter demonstrate that it is usually a single vessel descending from the rostral cerebral artery. The vessel, having passed the lateral olfactory sulcus, gets divided along its course into respective branches, its main trunk runs towards the fornix of the brain. In sheep such a middle cerebral artery division pattern was reported in $93.3 \%$ of the cases. In that species there were found cases of the descent, from the rostral cerebral artery, of two independent branches of the middle cerebral artery in $6.7 \%$ of the cases. In other mammal species a double descent of the middle cerebral artery from the rostral cerebral artery was reported at various percentages. Most often it was recorded in wild boar in 62\% (Skoczylas \& Wiland 1999), in domestic pig 38.7\% (Skoczylas 2000), in moose in $6.25 \%$ of the cases (Jabłoński et al. 1999a). According to Wiland \& Brudnicki (1984), multiple middle cerebral arteries occur in domestic pig and wild boar and in other mammal species they are often described as vascular variations.

\section{CONCLUSIONS}

There was variability in the studied vascular area in sheep. In sheep the anterior olfactory artery in $30 \%$ of the cases investigated was a vessel which descended independently from the main trunk of the middle cerebral artery.

In that species there were found cases of the descent, from the rostral cerebral artery, of two independent branches of the middle cerebral artery in $6.7 \%$ of the cases.

In sheep, similarly as in Artiodactyla species investigated, the parietal branches are least developed.

Conflict of interest statement.- The authors have no competing interests.

\section{REFERENCES}

Baldwin B.A.1964. The anatomy of the arterial supply to the cranial regions of the sheep and ox. Am. J. Anat.115(1):101-108. <http://dx.doi.org/10.1002/ aja.1001150107><PMid:14201080>

Baldwin B.A. \& Bell F.R. 1963. The anatomy of the cerebral circulation of the sheep and ox. The dynamic distribution of the blood supplied by the carotid and vertebral arteries to cranial regions. J. Anat. 97:203-216. <PMid:13969329>

Brauer K. \& Schaber W. 1970. Album of Brain in Mammals. VEB Gustav Fisher Verlag, Jena.

Brudnicki W., Jabłoński R., Nowicki W. \& Skoczylas B. 2005. Cortical branches of the middle cerebral artery in goat (Capra hircus). Bydgoszcz Scientific Society, Committee on Agricultural and Biological Sciences, Bydgoszcz B, 56:29-32.

Chadzypanagiotis D. 1975. Arteries on the surface of the cerebral hemisphere in the cat. Folia Morphologica, Warsaw 32(4):385-399. <PMid:4548682>

Jabłoński R. \& Wiland C. 1973. Variation of the arteries of the base of the brain in sheep. Folia Morphologica, Warsaw 32(3):339-347. <PMid:4543327>

Jabłoński R. \& Roskosz T. 1997. Middle cerebral artery (a. cerebri media) in roe-deer (Capreolus capreolus, L.). Annals Warsaw Agricult. Univ. SGGW. Vet. Med. 20:35-41.

Jabłoński R., Skoczylas B. \& Wiland C. 1999a. The main branches of the middle cerebral artery in elk (Alces alces). Electron. J. Polish Agricult. Univ. Vet. Med. 2(2):1-6.

Jabłoński R., Kubica I., Skoczylas B. \& Wiland C. 1999b. Cortical branches of the middle cerebral artery in cattle (Bos primigenius f. dom.). ATR Scient. J. 219. Animal Husbandry 30:47-54.

Skoczylas B. 2000. Cortical branches of middle cerebral artery in domestic pig (Sus scrofaf. domestica). Electron. J. Polish Agricult. Univ. Vet. Med. 3(1)

Skoczylas B. \& Wiland C. 1999. Cortical branches of the middle cerebral artery in the wild boar. (Sus scrofa L.). Electron. J. Polish Agricult. Univ. Vet. Med. 2(1/6)

Skoczylas B., Brudnicki W., Nowicki W., Jabłoński R., Kirkiłło-Stacewicz K. \& Wach J. 2011. Cortical branches of the middle cerebral artery in fallow deer (Dama dama). Electron.J. Polish Agricult. Univ. Vet. Med. 14(4):9.

Skoczylas B., Brudnicki W., Nowicki W., Kirkiłło-Stacewicz K., Jabloński R. \& Wach J. 2012. The cortical branches of the middle cerebral artery in the otter (Lutra lutra). Veterinarni Medicina 57(6):282-286. <http://dx.doi. org/10.17221/6014-VETMED>

Walinczus J. 1973. The middle cerebral artery in domestic pig. Scient. Materials Vet. Inst. Witebsk 26:123-127.

Węgrzyn M., Roskosz T. \& Mazowiecka M. 1983. Brain arteries of the European bison (Bison bonasus L.,1758). Annals Warsaw Agricult. Univ. SGGW-AR. Vet. Med. 11:9-16.

Wiland C. 1991. Comparative investigation of cortical branches of middle cerebral artery in some species of carnivores. ATR Scient. J., Bydgoszcz, 44:1-52.

Wiland C. \& Brudnicki W. 1984. Multiple middle cerebral arteries in various species of mammals. Folia Morphologica, Warsaw 53(3):265-270. <PMid:6335479> 